\title{
Utilization of standardized patients and case studies to evaluate effect of SBIRT training for APRN's
}

\author{
Deborah MacMillan ${ }^{1 *}$, Sheryl Winn ${ }^{1}$, Sallie Coke ${ }^{1}$, Annie Biers $^{2}$, Sylvia Shellenberger ${ }^{2}$ \\ From INEBRIA 12th Congress, \\ Atlanta, GA, USA. 24-25 September 2015
}

\section{Background}

Research supports the efficacy of screening, brief intervention, and referral to treatment (SBIRT) in reducing unhealthy substance use, and demonstrates that nurses are effective SBIRT providers. The purpose of utilizing MI is to help clients to manage their health independently, while building their confidence and willingness to change behavior [1]. Identifying effective pedagogical methods for teaching and evaluating students' proficiency in utilizing SBIRT is essential.

\section{Methods}

Family Nurse Practitioner (FNP) students $(\mathrm{n}=36)$ received SBIRT and Motivational Interviewing (MI) didactic content and clinical practice during three consecutive semesters. The students' skills were assessed during the $1^{\text {st }}$ semester (Time One) and the $3^{\text {rd }}$ semester (Time Two) using standardized patient case scenarios. Student encounters were recorded and evaluated by faculty, standardized patients and self-reflections. Debriefing sessions provided face to face feedback by faculty and MI trainers.

\section{Results}

The percentage of students who were able to demonstrate six specific SBIRT skills increased from Time One measure to Time Two measure in four areas: identifying risk ( $82.6 \%$ vs.100\%), educating on low risk drinking limits $(67.4 \%$ vs. $97.2 \%)$, recommendation to quit or cut back (78.3\% vs. $97.2 \%)$, and enhancing motivation (67.4\% vs. $77.8 \%)$. The remaining two areas showed a

\footnotetext{
* Correspondence: debby.macmillan@gcsu.edu

'School of Nursing, Georgia College \& State University, Milledgeville, Georgia 31061, USA

Full list of author information is available at the end of the article
}

slight decrease in the percentage of students who demonstrated those skills: asking permission to raise the subject (97.8\% to $94.4 \%$ ) and explaining the connection between substance use and the reason(s) for patient's current medical visit $(89.1 \%$ vs. $88.9 \%)$. Student's selfreflections accurately identified areas of strengths and weakness in their individual SBIRT skills and were consistent with faculty evaluations of their performance.

\section{Conclusion}

The use of standardized patient case scenarios was shown to be an effective venue for faculty to evaluate student proficiency at performing SBIRT. Educating the FNP student in SBIRT and MI will result in more clients being screened and treated for alcohol or drug misuse and to empower these clients to independently manage their health with success.

\section{Authors' details}

'School of Nursing, Georgia College \& State University, Milledgeville, Georgia 31061, USA. ${ }^{2}$ School of Medicine, Mercer University, Macon, Georgia 31206, USA.

\section{Published: 24 September 2015}

\section{References}

1. Czart M: Using 3D virtual standardized patient to teach motivational interviewing. Journal of Virtual Worlds Research 2014, 7(2):1-22.

2. Ebbert DW, Connors H: Standardized patient experiences; evaluation of clinical performance and nurse practitioner student satisfaction. Nursing Education Perspectives 2004, 25(1):12-15.

3. Rutherford-Hemming T, Jennrich JA: Using standardized patients to strengthen nurse practitioner competency in the clinical setting. Nursing Education Perspectives 2013, 34(2):118-121.

\section{doi:10.1186/1940-0640-10-S2-031}

Cite this article as: MacMillan et al:: Utilization of standardized patients and case studies to evaluate effect of SBIRT training for APRN's. Addiction Science \& Clinical Practice 2015 10(Suppl 2):O31. 